\title{
A Characterization of Ultrasonic Full Angle Spatial Compounding as a Possible Alternative for Breast Cancer Screening
}

\author{
Luis MEDINA, Jorge CAMACHO, Carlos FRITSCH \\ Group of Ultrasonic Systems and Technology (GSTU-ITEFI) \\ Spanish National Research Council (CSIC) \\ Serrano, 144, 28006 Madrid, Spain; e-mail: luis.medina@csic.es \\ (received September 1, 2014; accepted May 21, 2015)
}

\begin{abstract}
Breast cancer screening is based on X-ray mammography, while ultrasound is considered a complementary technique with improved detection in dense tissue. However, breast cancer screening requires a technique that provides repeatable results at the inspection interval which cannot be achieved with manual breast exploration.

During the last years there have appeared several approaches to overcome this limitation by means of automated ultrasonic tomography performed with motorized probes or with a large set of array transducers.

This work addresses these problems by considering a quite simple and low-cost arrangement, formed with a ring of conventional medical-grade array probes which are multiplexed to the electronics to build Full Angle Spatially Compounded (FASC) images. The work analyzes the performance of such arrangement in terms of resolution and isotropy, showing by numerical modelling and experimentally that it provides high resolution and homogeneity in the whole imaged region.

The implementation of this technique would provide more than one circular FASC per second and a whole breast volume image in 1-2 minutes with conventional technology, a process fast enough to be clinically useful. Moreover, the automated technique is repeatable and can be used by the clinician to perform immediately the diagnosis without requiring additional data processing.
\end{abstract}

Keywords: breast cancer, Full Angle Spatial Compounding, resolution, isotropy.

\section{Introduction}

Current practices for breast cancer screening are based on mammography, which uses ionizing radiation, while ultrasound is considered a complementary technique in the further assessment of both palpable and impalpable breast abnormalities (TEH, WILSON, 1998). However, it has been found that ultrasound provides improved breast cancer detection for mammographically dense breast tissue (NothACKer et al., 2009). Furthermore, breast cancer is a lobar disease that is spatially localized, arising in most cases within a single ductal tree (Тот, 2011). This precludes early stage detection from mammograms since they do not provide the required fine spatial resolution in the order of 0.1 to $0.3 \mathrm{~mm}$, as diagnoses are performed in mammograms by the appreciation of some local increase of density over time. By contrast, some ultrasound techniques, such as Ductal Echography, have proved to overcome this problem by directly imaging the ductal-lobular structures using high-frequency scanners (TeBoul, 1998; 2010). Although Ductal Echography provides accurate diagnosis, this technique also has some drawbacks. First, the long time it takes to scan manually the breast to find the lobe distribution surrounding the nipple. Second, the lack of accurate positioning that makes it difficult to track the findings over time. To overcome these drawbacks, automated radial breast scanning has been proposed (ROUYER et al., 2010; GoOding et al., 2010).

For ultrasound techniques to be useful to breast cancer screening, a different approach has to be followed. Besides repeatable image positioning and high resolution and contrast, short time to perform the exploration is required to provide the clinician with a real-time display. This is important for screening, since it will be applied to the large population of asymptomatic women, so that only a few minutes are available for a global exploration unless suspicious indications are found. This suggests that fast, automated 
scanning with limited processing burden is advisable for this purpose.

During the last years an increasing interest in the development of ultrasound computed tomography (USCT) for breast cancer detection has been observed. The proposed methods emulate the X-ray Computed Tomography (CT) techniques, using an emitter and receiver in opposite sides to obtain attenuation and velocity maps using single element (CHENEvERT et al., 1984) or array transducers (STOZKA et al., 2002; WaAg, Fedewa, 2006; Quan, Huang, 2007; MarMARELIS et al., 2007; WISKIN et al., 2010; ROY et al., 2013). These are automated approaches that provide tomograms at different coronal planes or whole volume images.

However, differently from X-ray CT, ultrasonic rays do not propagate in straight trajectories in biological tissue due to refraction (variations in the propagation velocity) and diffraction (scatterer distribution). Therefore, to get accurate enough speed and attenuation maps, iterative procedures must be followed solving the inverse and direct problems until equilibrium is reached. These are intensive computing algorithms that need to be implemented in high performance hardware to achieve results within a reasonable time (DAPP et al., 2012).

On the other hand, the Multi-Angle Compounding Imaging (MACI) creates a single image by averaging several individual linear-scan B-mode images acquired with a diversity of angular directions (HILLER et al., 1984; Dines et al., 1987). It has been shown that MACI improves resolution, contrast, and speckleto-noise ratio (SNR) and reduces angle-dependent artifacts (ENTREKIN, 1999). MACI is usually achieved with a small set of steering angles, ranging typically below $\pm 20^{\circ}$ to preserve the depth of the fully compounded region (JESPERSEN, 1998). Nonlinear operators such as root mean square, geometrical mean and maximum, have been also proposed to suppress strong echoes at the expense of some losses on SNR (WilHJELm et al., 2004). In any case, it has been found that image quality in general improves with larger angular diversity, as spatial uncorrelation is increased.

More recently tomograms are being formed by composing sector B-scan images over $360^{\circ}$, a technique known as Full Angle Spatially Compounding (FASC) (HANSEn, 2008). It has been shown that FASC images have increased resolution and contrast and at the same time avoid many angle-dependent artifacts.

FASC has further advantages. First, automated systems can be implemented by mechanical rotation of a phased array probe around the breast (HANSEN et al., 2008; Wiskin et al., 2010; ROUYER et al., 2010) or a transducer arrangement with synthetic aperture reconstruction following data acquisition (STOZTKA et al., 2002; Ruiter et al., 2012). Second, processing burden is low since individual B-scan images are ob- tained in real-time and the compounding process can be achieved simultaneously with adequate scan conversion algorithms. Third, most of the technology is conventional and readily available. This opens an opportunity to build tomograms fast enough to be considered a technique for breast cancer screening alternative to mammography.

To this aim, a simple and moderate cost system will use a set of clinical grade array probes uniformly distributed in a circular arrangement that are multiplexed to a conventional scanner performing FASC (Fig. 1). This is considered much less expensive that designing dense ring array transducers and much faster than using a single array probe mechanically rotated. The effect of the sparse transducer on the FASC image quality can be very low if every individual array verifies the $0.5 \lambda$ pitch to avoid grating lobes. In fact, FASC images are formed from a set of images acquired at discrete angular positions or Single Angle Images (SAIs).

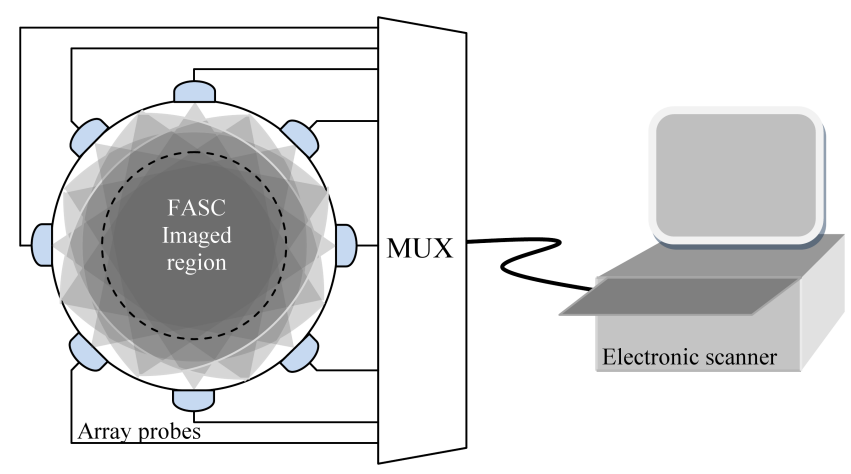

Fig. 1. Schematic to perform FASC at high speed and low cost.

On the other hand, this arrangement would allow multi-modality imaging including reflectivity, attenuation, and velocity maps (СAMACHO et al. 2012). However, to shorten the diagnosis time, a rapid exploration would be first achieved obtaining high-resolution FASC tomograms. If suspicious indications are found, the system could be switched to other computing intensive modalities to provide further diagnosis information to assess the malignancy of the lesions.

This process can be performed for the whole breast volume at a low cost and in a short time. A conventional echograph obtains a B-scan sector image typically in less than $1 / 24 \mathrm{~s}$, so that a FASC tomogram compounding up to $24 \mathrm{~B}$-scan sector images could be produced in less than $1 \mathrm{~s}$. Taking several (i.e., 60) FASC tomograms along $120 \mathrm{~mm}$ of the coronal axis at $2 \mathrm{~mm}$ intervals would be achieved in less than 1 minute, which is a time interval clinically quite acceptable.

Differently from mammography, where a single projection is obtained, this procedure will provide the clin- 
ician with a 3D ultrasound image of the whole breast volume. Of course to further accelerate the diagnosis task, it would be also possible integrating the result of several tomograms in a single $2 \mathrm{D}$ image, like it is done with mammography, by simple image processing techniques such as averaging, mean, $\max / \mathrm{min}$, etc. 3D image segmentation would provide even improved diagnosis information.

However, differently from the MACI case, there are no analyses showing the quantitative improvements provided by FASC. In this paper we address the characterization of a FASC system aimed to provide fast reflection tomograms using a set of standard phased array probes. Our interest is investigating the expected resolution, isotropy, and homogeneity in the imaged region as a function of the number $M$ of compounded images which affects the hardware requirements. The work is carried out first by a numerical model to obtain the compounded Point Spread Function (PSF), which is then experimentally validated with a first prototype where a single array probe is rotated around the region of interest (ROI), emulating the final system with multiple arrays. Conclusions regarding the minimum hardware requirements and the expected performances are drawn.

\section{Methodology}

The aim of this work is to get quantitative information of the FASC resolution, isotropy, and homogeneity in the whole imaged region. The FASC image is composed from $M$ Single-Angle Images (SAIs) that are conventional sector B-scans taken at discrete angular positions $\Phi$ in a $360^{\circ}$ circular trajectory with a radius $R$ (Fig. 2).

A numerical method based on a simplified PSF model is followed. In this work resolution is defined

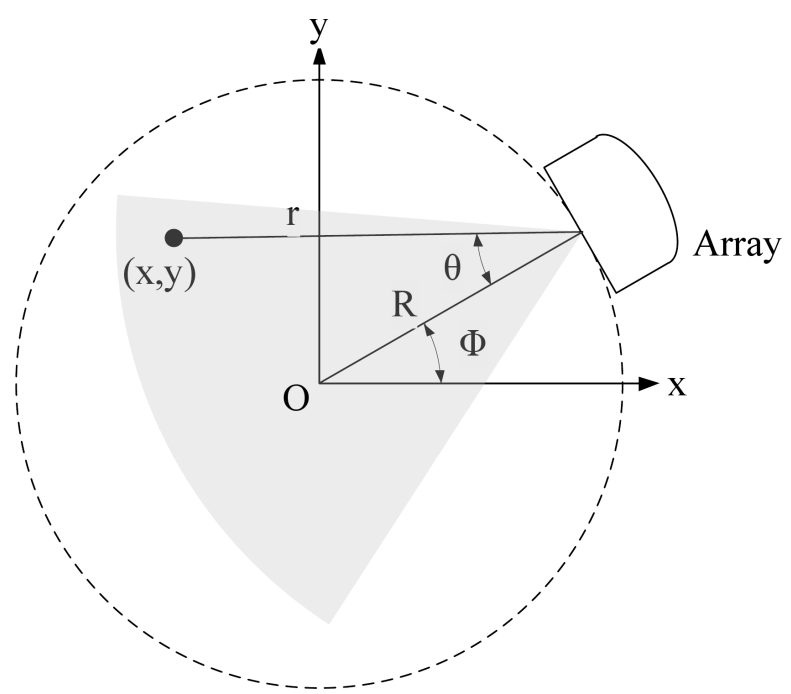

Fig. 2. Geometry for Full Angle Spatial Compounding (FASC). by the Full Width at Half Maximum (FWHM), which yields the PSF extent at $-6 \mathrm{~dB}$ from its maximum. It is also assumed that the SAI PSF is separable into radial $A_{r}$ and angular $A_{\theta}$ components:

$$
P S F\left(r, t, \theta, \theta_{f}\right)=A_{r}(r, t) A_{\theta}\left(\theta, \theta_{f}\right),
$$

where $r$ is radius, $\theta$ is angular coordinate, $t$ is time and $\theta_{f}$ is the steering angle within the sector image, all referred to the aperture centre. This is the usual assumption, where the radial and angular components are responsible for the axial and lateral resolution, respectively.

The ultrasonic signal is modeled as a Gaussian pulse:

$$
u(t)=e^{-t^{2} / 2 b^{2}} \cos (\omega t),
$$

where $\omega$ is the ultrasound angular frequency and $b$ is a parameter inversely proportional to the fractional bandwidth $B$ :

$$
b=\frac{\sqrt{8 \cdot \ln 2}}{\omega B} .
$$

The acoustic wave presents losses due to attenuation and propagation:

$$
l_{n}(r)=\frac{e^{-\alpha r}}{r},
$$

where $\alpha$ represents the attenuation coefficient at the frequency of interest.

The model for the radial component of the PSF is given by the product of (2) and (4). Using the equivalence in pulse-echo imaging $t=2 r / c$, where $c$ is propagation velocity and $\lambda=2 \pi c / \omega$, the radial component $A_{r}(r)$ is:

$$
A_{r}(r)=e^{-2 r^{2} / c^{2} b^{2}} \cos \left(\frac{4 \pi r}{\lambda}\right) \frac{e^{-2 \alpha r}}{2 r} .
$$

The SAI axial resolution at a given range or FWHM of the PSF radial component can be found equating the envelope of (5) to half its maximum value. Assuming that the attenuation term is constant in the FWHM interval, it yields

$$
\Delta r_{S A I}=2 \ln 2 \frac{\lambda}{\pi B} .
$$

On the other hand, at focus, the main lobe is practically equal for continuous wave and for wideband excitations, being their main differences in the side lobes. Thus, the simplified model for the angular component can be approximated by the continuous wave array factor $F$ between zeroes (STEINBERG, 1976):

$$
F(\theta)=\left|\frac{\sin \left[\frac{\pi D}{\lambda}\left(\sin \theta_{f}-\sin \theta\right)\right]}{\sin \left[\frac{\pi d}{\lambda}\left(\sin \theta_{f}-\sin \theta\right)\right]}\right|
$$

where $D=N \cdot d$ is the aperture size, with $N$ being the number of array elements and $d$ being the element 
pitch. The main lobe is between the two first zeroes of $F$ with an angular extent:

$$
\Delta \theta_{Z} \approx 2 \frac{\lambda}{D \cos \theta_{f}} .
$$

The angular FWHM is usually considered half the above value, $\Delta \theta_{S A I}=\Delta \theta_{Z} / 2$ and the lateral resolution depends on the range:

$$
\Delta l_{S A I}=r \Delta \theta_{S A I} .
$$

This is the cause of the resolution anisotropy of conventional B-mode images, where axial and lateral resolutions are not directly correlated (Eqs. (6) and (9)).

The model above considers that elements are points and, thus, omnidirectional. However, real array elements have finite dimensions whose diffraction effects reduce their sensitivity with the steering angle. The normalized amplitude for a single element in pulseecho is (KINO, 2000):

$$
A\left(\theta_{f}\right)=\left|\operatorname{jinc}^{2}\left(\frac{d-g}{\lambda} \sin \left(\theta_{f}\right)\right)\right|,
$$

where $g$ is the gap between elements $(\operatorname{kerf}), \operatorname{jinc}(x)=$ $\mathrm{J}_{1}(2 \pi x) /(\pi x)$, and $\mathrm{J}_{1}(x)$ is the first-order Bessel function. A more realistic model for the PSF angular component $A_{\theta}\left(\theta, \theta_{f}\right)$ is given by the product of (7) and (10):

$$
\begin{aligned}
A_{\theta}\left(\theta, \theta_{f}\right)= & \left|\frac{\sin \left[\frac{\pi D}{\lambda}\left(\sin \theta_{f}-\sin \theta\right)\right]}{\sin \left[\frac{\pi d}{\lambda}\left(\sin \theta_{f}-\sin \theta\right)\right]}\right| \\
& \cdot\left|\operatorname{jinc}^{2}\left(\frac{d-g}{\lambda} \sin \left(\theta_{f}\right)\right)\right|
\end{aligned}
$$

The product of (5) and (11) yields the PSF, $P(r, \theta)$ at the point $(r, \theta)$ in the local coordinates as a function of array parameters $N, d, g, B, \lambda$, and propagation constants $c$ and $\alpha$.

The Compounded Point Spread Function, CPSF, is obtained by averaging the individual PSFs of the SAIs. For this purpose, a grid is set in absolute Cartesian coordinates with the origin arbitrarily located at the image centre. A grid point $\mathrm{P}$ represented by the vector $\mathbf{p}=(x, y, 0)$ has a corresponding point in the local coordinates $(r, \theta)$ of the array located at $(R, \Phi)$, where $R$ is the ring radius and $\Phi$ is the array steering angle (see Fig. 2). The normal to the array is represented by the unit vector $\mathbf{n}=(-\cos \Phi, \sin \Phi, 0)$ and the angle $\theta$ can be determined by the cross product of the normal with the vector $\mathbf{r}$ from the array centre at $(R \cos \Phi$, $R \sin \Phi, 0)$ to the point $\mathrm{P}$, that is, $\mathbf{r}=(x-R \cos \Phi, y-$ $R \sin \Phi, 0) /|\mathbf{r}|$ :

$$
\begin{aligned}
\sin \theta & =|\mathbf{n} \times \mathbf{r}|, \\
r & =\sqrt{(x-R \cos \Phi)^{2}+(y-R \sin \Phi)^{2}} .
\end{aligned}
$$

These equations yield the local coordinates $(r, \theta)$ of a pixel at $(x, y)$ where the individual PSFs are computed for the $M$ array positions. Then, all values are averaged to obtain the CPSF.

Note that other functions, different from the mean, can be used to get the theoretical CPSF as suggested in (WilhJelm et al., 2004). The simplified model is general for Gaussian shaped ultrasonic pulses and can be adapted for different array frequencies, bandwidth, and aperture size.

\section{Materials and methods}

In this study the ring of transducers is emulated by rotating $360^{\circ}$ a single array probe with a nominal radius $R=95 \mathrm{~mm}$ inside a cylindrical water tank where the targets are positioned. Movement is provided by stepper motors and a worm-wheel attachment with a high angular positioning accuracy. Both the arrangement and the required calibration procedure were previously described in (CAMACHO et al., 2012).

The single probe was a medical grade 128element, $3.2 \mathrm{MHz}$ phased array with $d=0.22 \mathrm{~mm}$, relative bandwidth of $50 \%$, and elevation focus at $75 \mathrm{~mm}$ (Prosonic, Korea). Images were acquired with a SITAU-112 instrument (Dasel, Spain), with 128 fullparallel active channels, no focusing in emission and dynamic focus in reception.

A stainless steel $0.26 \mathrm{~mm}$ diameter wire target was used to experimentally verify image resolution, sensitivity, and isotropy. It was located at different positions, from the centre of the ROI to the periphery at $10 \mathrm{~mm}$ intervals on the $x$-axis (Table 1 ). Because of the system circular symmetry, this set of positions is sufficient to calculate the PSF at practically any arbitrary point into the ROI.

Table 1. Absolute coordinates of the wire target found experimentally.

\begin{tabular}{|c|c|c|}
\hline Position & $x[\mathrm{~mm}]$ & $y[\mathrm{~mm}]$ \\
\hline 1 & -0.6 & -0.1 \\
\hline 2 & -10.0 & 0.1 \\
\hline 3 & -19.9 & 0.15 \\
\hline 4 & -30.2 & 0.1 \\
\hline 5 & -40.0 & 0.1 \\
\hline 6 & -50.45 & 0.25 \\
\hline 7 & -60.3 & 0.15 \\
\hline
\end{tabular}

For each position of the point reflector, a full set of 360 sector images (SAIs) were acquired at $\Delta \Phi=1^{\circ}$ intervals. To reduce memory resources and computation time, each SAI was a $\theta= \pm 5^{\circ}$ sector scan centred at the reflector position, with the angular step $\Delta \theta=0.1^{\circ}$ and range $r= \pm 5 \mathrm{~mm}$, sampled at $40 \mathrm{Msps}$ and 12 bits. The image size is sufficient to fit the main 
lobe of individual PSFs, and oversampling in axial and lateral directions ensure accurate results without further interpolation. Each image line was beamformed with unfocused emission and strict dynamic focusing reception (a focus per output sample). These operations were carried out automatically by the instrument in real time. The acquired images were sent to an external PC through an USB 2.0 link for further processing in MATLAB (The MathWorks, USA).

The image compounding process averages the envelope-detected B-scan images, previously interpolated on a Cartesian grid that covers the ROI. This way, the CPSF was obtained for each reflector position using subsets of $M=8$ and 36 angular positions in the acquired dataset. The CPSF at ROI points outside the $x$-axis were evaluated by angular displacing those subsets instead of changing the reflector position, which is possible due to the system angular symmetry.

\section{Results}

Theoretical (numerical model) and experimental (measured) CPSF images of point reflectors have been obtained and compared to assess the validity of the model. The numerical model was programmed with the experimental arrangement and array parameters, specifically, with the $(x, y)$ coordinates of the wire target that were experimentally obtained by locating the maximum of the SAI PSF.

Figure 3 compares the theoretical (left) and experimental (right) individual PSFs of the image taken at $\Phi=130^{\circ}$ of the target located at $(-50.45,0.25) \mathrm{mm}$ in the rectangular grid. Differences in the PSF width can be explained by the finite diameter of the "point reflector" used in the experiments $(0.26 \mathrm{~mm})$, which widens the PSF of an ideal point reflector. This is confirmed by the graph in Fig. 4 which compares the theoretical
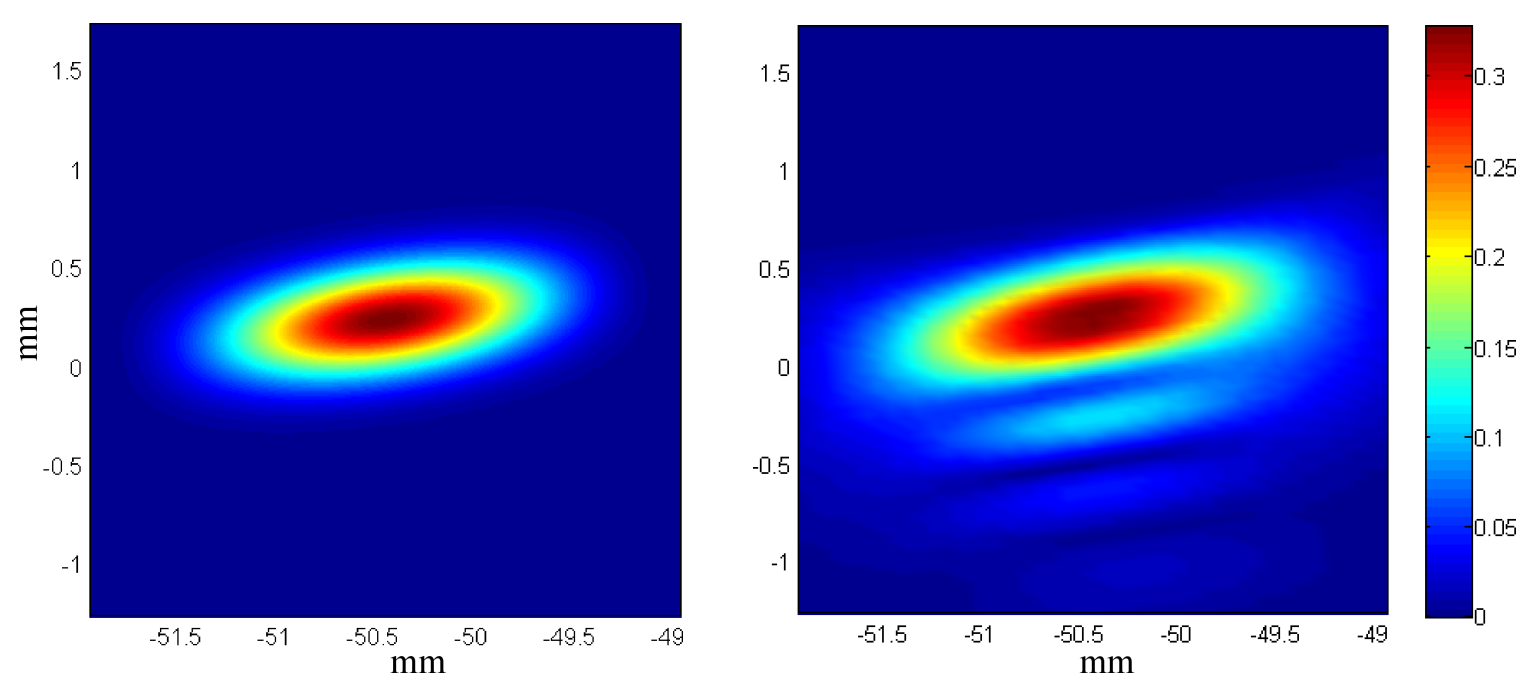

Fig. 3. Left: Theoretical PSF of a point target at $(-50.45,0.25)$. Right: experimental image.
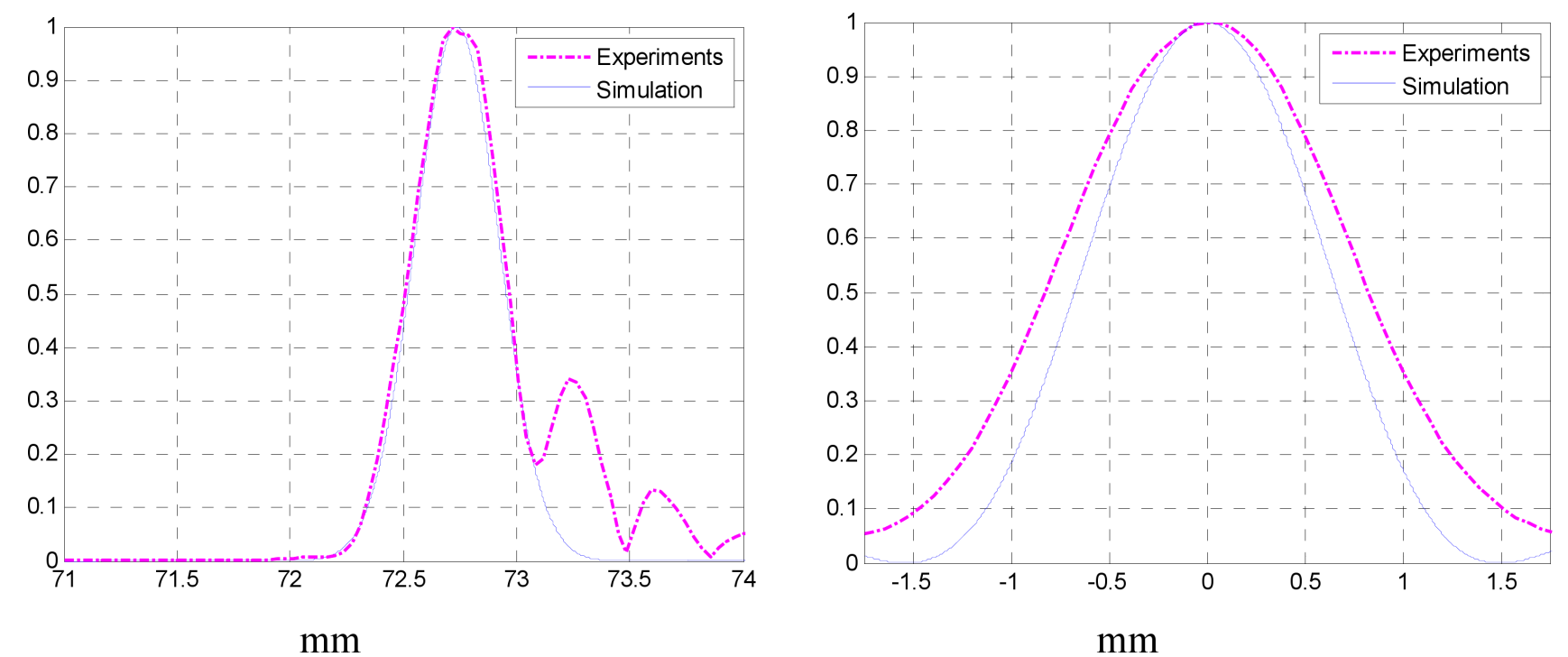

Fig. 4. Left: Axial patterns of the experimental (dash-dot) and theoretical (solid) images.

Right: Lateral patterns. Normalized amplitude. 
and experimental axial and lateral beam patterns. No difference is observed in the $-6 \mathrm{~dB}$ length in the axial direction because the same wire target was used to characterize the transducer's bandwidth. By contrast, in the lateral direction, the FWHM of the experimental main lobe is $0.3 \mathrm{~mm}$ wider than the theoretical one that considers a point reflector, consistent with the target diameter of $0.26 \mathrm{~mm}$. The theoretical axial and lateral resolution values agree with those expected from Eq. (6) and Eq. (11).

The experimental image shows some secondary echoes that are produced by creeping waves surrounding the wire target. Fortunately, these artifacts do not affect axial and lateral resolution measurements, which agree reasonably well at $-6 \mathrm{~dB}$ as shown in Fig. 4 . The high resolution anisotropy in both cases is characteristic of B-mode images.

The CPSF for this wire position and $M=36$ is shown in Fig. 5. The improvements in resolution and isotropy are evident. The decrease of the maximum amplitude of the experimental CPSF is due to saturation when the target went located near the array probe. The halo in the experimental image is caused by creeping waves surrounding the wire target, although it does not affect resolution measurement at $-6 \mathrm{~dB}$.

To quantitatively characterize the image, the following definitions are used with reference to the image centre for both the theoretical and experimental CPSF images:

a) Radial resolution $(\Delta r)$ : FWHM of the CPSF in the radial direction $(\mathrm{mm})$.

b) Angular resolution $(\Delta \varphi)$ : FWHM of the CPSF in the angular direction (rad).

c) Lateral resolution $(\Delta t)$ : length of the arc with an angle equal to the angular resolution with the radius $\rho=\sqrt{x^{2}+y^{2}}$, that is, $\Delta t=\rho \cdot \Delta \varphi(\mathrm{mm})$.

d) Isotropy $(\mu)$ : ratio of the radial to tangential resolution, $\mu=\Delta r / \Delta t$. Ideally, $\mu=1$, being a quan- titative figure of the variations of resolution with direction.

Application of these definitions to the experimental CPSF of Fig. 5 results in a similar radial and tangential resolution ( 0.59 and $0.60 \mathrm{~mm}$, respectively), about a $50 \%$ higher than the axial resolution of $0.41 \mathrm{~mm}$ given by (6). Isotropy is 0.98 .

Conversely, the experimental CPSF for $M=8$ SAIs is shown in Fig. 6. Slight homogeneity losses are observed, although the main lobe above $-6 \mathrm{~dB}$ is kept, as the radial and tangential resolution values confirm ( $0.60 \mathrm{~mm}$ and $0.63 \mathrm{~mm}$, respectively), close to those found for $M=36$. However, reducing $M$ to 8 increases image artifacts due to side-lobes.

It is interesting to analyze if these numbers can be generalized to the whole imaged region. The theoretical (left) and experimental (right) maps of $\Delta r, \Delta t$, and $\mu$ are presented in Figs. 7 to 9 for a composition of $M=8$ SAIs, which can be considered a lower bound for image compounding. The color scales are common to both theoretical and experimental maps. Several points must be highlighted with reference to the experimental data:

1. Resolution (radial in Fig. 7 and lateral in Fig. 8) remains nearly constant in the whole image, especially in the central region within a radius of $40 \mathrm{~mm}$, in spite of the theoretical and experimental distributions appearing dissimilar in the imaged maps. The radial resolution is between 0.58 and $0.63 \mathrm{~mm}$ and between 0.6 and $0.68 \mathrm{~mm}$ in the theoretical and experimental maps, respectively. The lateral resolution is between 0.61 and $0.65 \mathrm{~mm}$ and between 0.58 and $0.68 \mathrm{~mm}$ in the theoretical and experimental maps, respectively. The resolution differences between the theoretical and experimental data are quite low, thus, validating the numerical model. Besides, these variations are significantly lower than those found with conventional B-mode images.
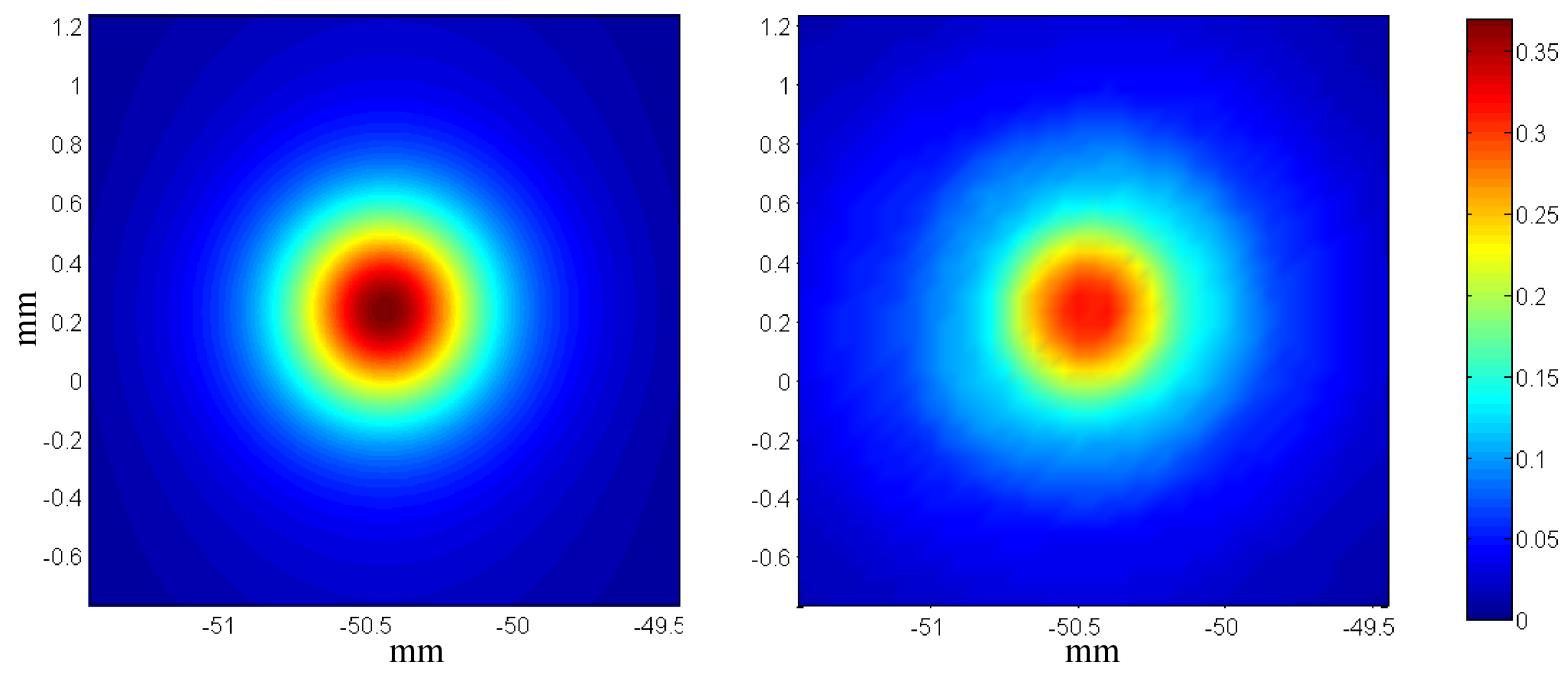

Fig. 5. CPSF of the point target at $(-50.45,0.25)$. Left: theoretical, right: experimental. 

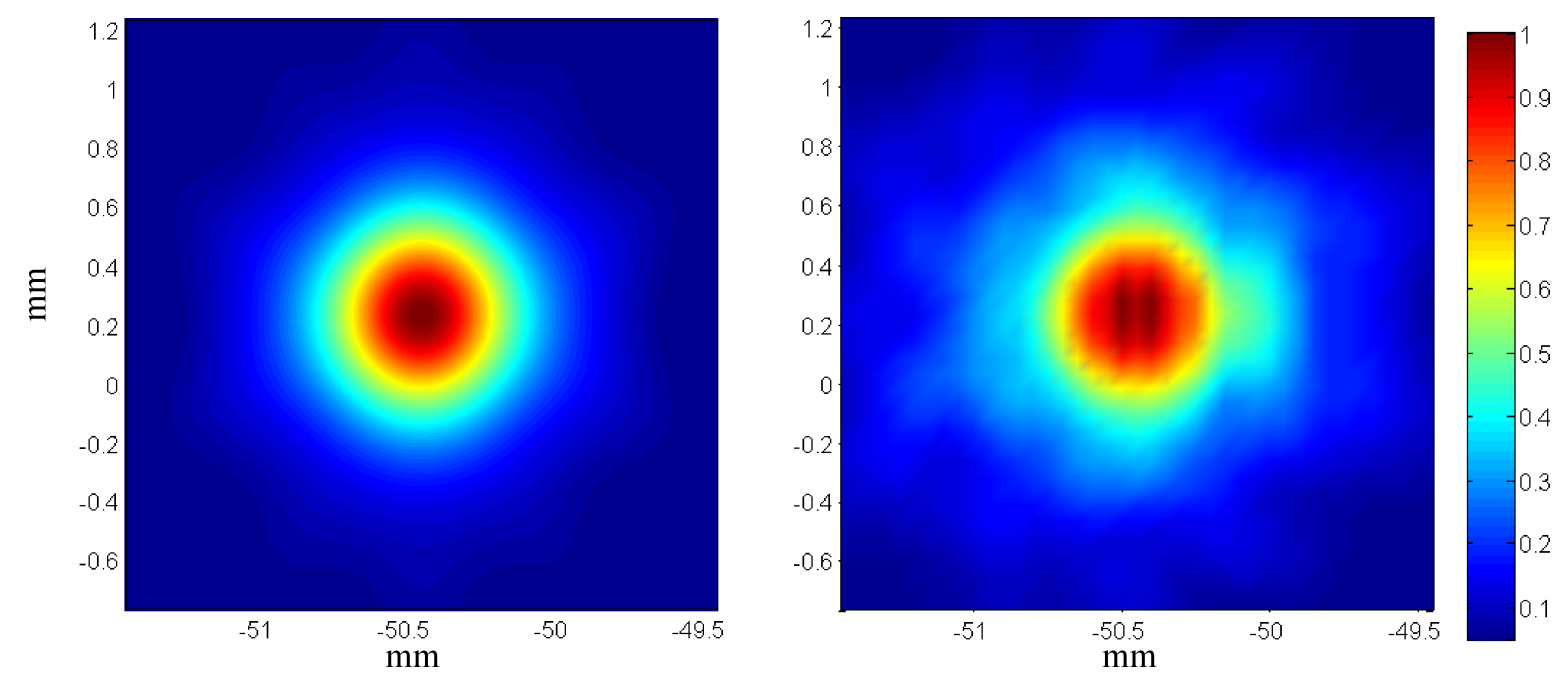

Fig. 6. CPSF of the point target at $(-50.45,0.25)$. Left: theoretical, right: experimental.
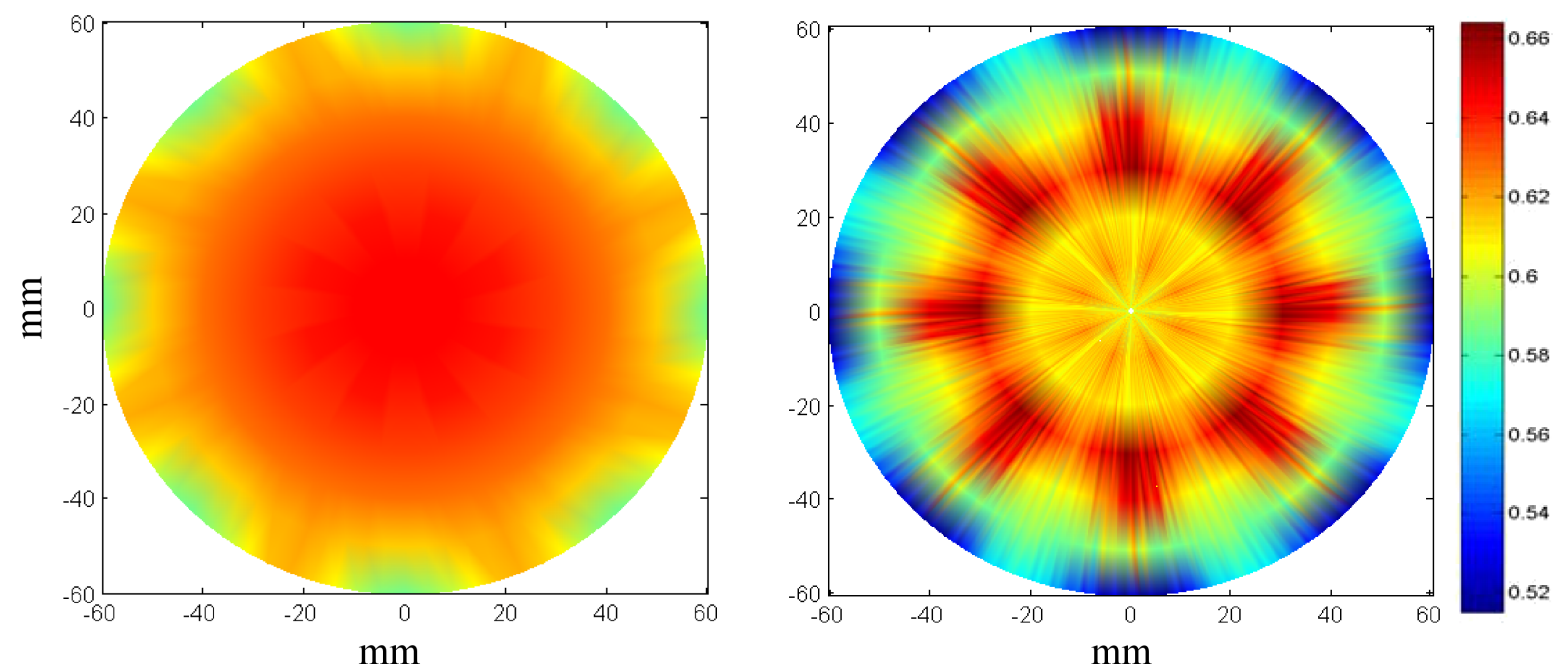

Fig. 7. Theoretical (left) and experimental (right) radial resolution maps for $M=8$ (mm).
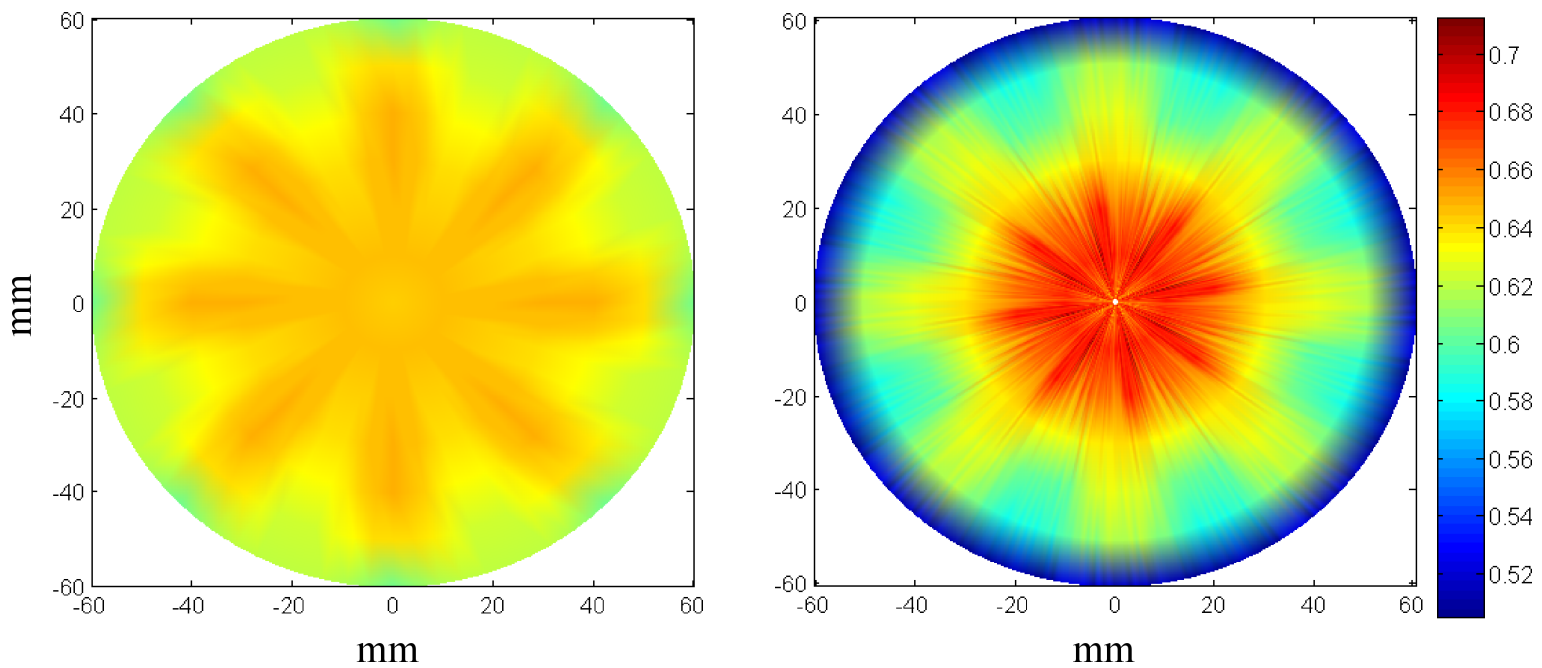

Fig. 8. Theoretical (left) and experimental (right) lateral resolution maps for $M=8(\mathrm{~mm})$. 

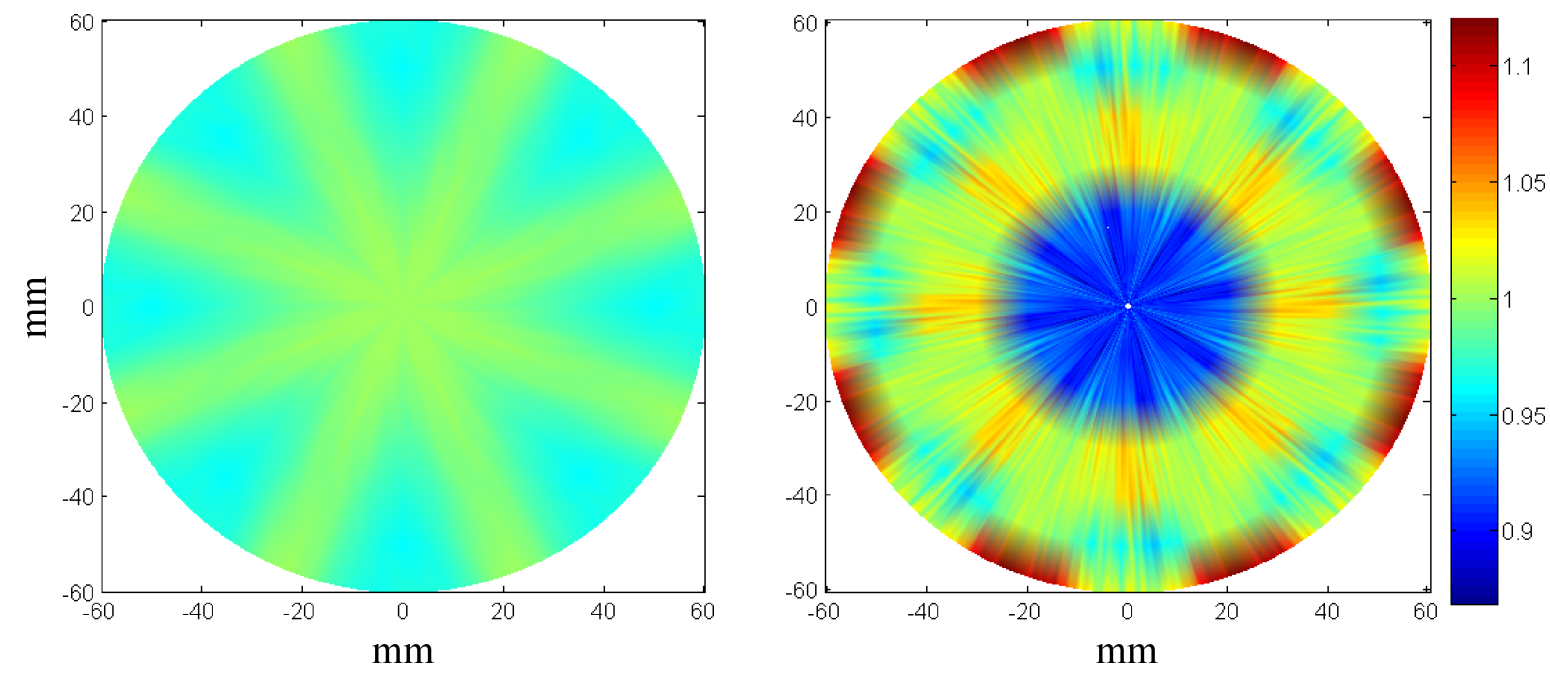

Fig. 9. Theoretical (left) and experimental (right) isotropy maps for $M=8$.

2. Image isotropy (Fig. 9) is a near unity in the whole image, ranging from 0.96 to 1.0 in the theoretical map and 0.85 to 1.12 in the experimental data. In spite of the variability difference, in both cases isotropy is near unity and approximately constant in the central region. This indicates again that the differentiation between the radial and lateral resolution is marginal, so that FASC images can be essentially described in terms of "resolution" (both are quite similar in absolute values, although their distribution in the image maps looks different).

3. Resolution slightly improves at a larger radius in both theoretical and experimental images. Although this counter intuitive effect can be ignored in practice due to the small variations found, it is explained by the better lateral resolution provided by the SAI B-mode images at lower ranges.

4. The low number of composed images in this case $(M=8)$ makes apparent the resolution variations as a function of angle (Figs. 7 and 8). The highest variability through the angular direction for $M=8$ found in the experimental images are below $4 \%$ for radial resolution, $2.5 \%$ for lateral resolution, and $3.5 \%$ for isotropy. In the whole image, the maximum variations are $\pm 12 \%$ and $\pm 15 \%$ for the radial and tangential resolutions, respectively.

5. For larger values of $M$, the variability of these parameters through the angular direction practically disappears. Figure 10 shows the radial and lateral resolution maps for $M=36$ (experimental data). In the central region, radial resolution varies between 0.6 and $0.63 \mathrm{~mm}( \pm 2.5 \%)$ and tangential resolution varies between 0.63 and $0.68 \mathrm{~mm}( \pm 4 \%)$ with the radius, confirming once again their similarity. These figures show that resolution is practically independent of the number of compounded images, at least as long as $M>8$.
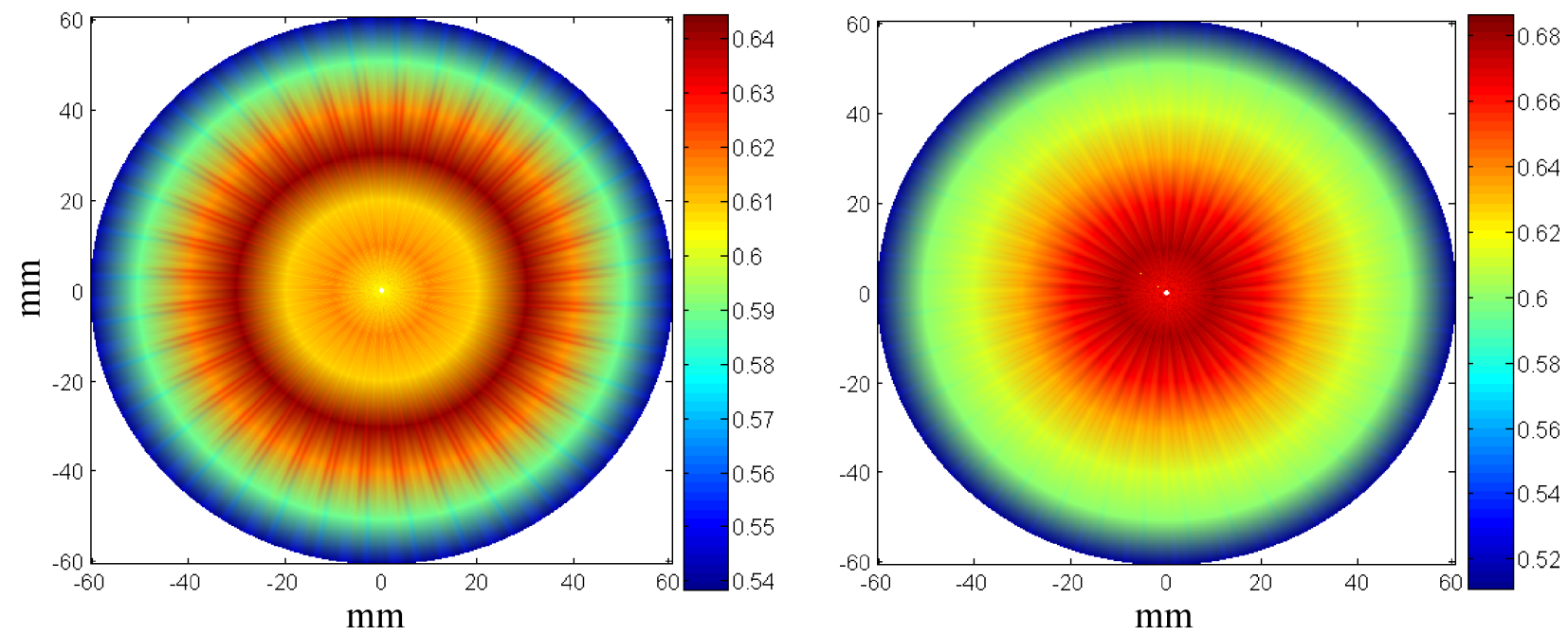

Fig. 10. Experimental resolution for $M=36$. Left: Radial; right: lateral. 
These results confirm that FASC images can be considered homogeneous with regard to resolution and isotropy (similar resolution at all ranges and directions). This is in contrast with the properties of standard B-mode images.

\section{Conclusion}

Full Angle Spatial Compounded (FASC) images have been analyzed both theoretically and experimentally for resolution, homogeneity, and isotropy. Although the theoretical and experimental maps look different, their numerical values are quite similar and, hence, experiments allow validating the proposed numerical model.

Resolution is practically constant and about 1.5 times the axial resolution provided by the transducer's bandwidth. Isotropy shows small variations around unity, so that a single term "resolution" can be applied to FASC images. It has been proved that these figures hold for an increasing number of compounded SAIs. In practice, compounding a small (i.e., $M=8$ ) number of Single Angle Images (SAIs) acquired from equidistant angular positions is enough to achieve these figures.

These results allow using a small set of $M$ array probes acquiring SAIs in a multiplexed mode to produce high quality FASC images at a high speed. Acquiring a set of FASC images at different heights would provide 3D volumetric images in a short time as well. Such a low-cost approach would achieve increased resolution, repeatability, and enough capabilities to be considered an alternative to the current x-ray based mammography for breast cancer screening.

\section{Acknowledgment}

This work has been supported by the project DPI2013-42236-R founded by the Spanish Ministry of Economy and Competitiveness and the project S2013/MIT-3024 funded by the Madrid Community and the EU.

\section{References}

1. Basoglu C., Kim Y., Chalana V. (1996), A realtime scan conversion algorithm on commercially available microprocessors, Ultras. Imag., 18, 241-260.

2. Camacho J., Medina L., Cruza J.F., Moreno J.M., FRITSCH C. (2012), Multimodal Ultrasonic Imaging for Breast Cancer Detection, Archives of Acoustics, 37, 3, 253-260.

3. Chenevert T., Bylsky D., Carson P., Meyer C., Bland P., Adler D., Schmitt R. (1984), Ultrasonic Computed Tomography of the Breast. Improvement of image quality by use of cross-correlation time-of-flight and phase-insensitive attenuation measurements, Radiology, 152, 155-159.
4. Dapp R., Gemmeke H., Ruiter N. (2011), 3D Refraction-Corrected Transmission Reconstruction for 3D Ultrasound Computer Tomography, Medical Imaging, Proc. SPIE, 8320, 832014 1-7.

5. Dines K., Goss S. (1987), Computed Ultrasonic Reflection Tomography, IEEE Trans. UFFC, 34, 3, 309318.

6. Entrekin R., Jackson P., Jago J.R., Porter B.A. (1999), Real Time Spatial Compound Imaging in breast ultrasound: technology and early clinical experience, Medicamundi, 43, 3, 35-43.

7. Gooding M., Finlay J., Shipley J., Halliwell M., Duck F. (2010), Three-Dimensional Ultrasound Imaging of Mammary Ducts in Lactating Women. A Feasibility Study, J. Ultrasound Med., 29, 95-103.

8. Hansen Ch., Hüttebräuker N., Hollenhorst M., Schasse A., Heuser L., Schulte-AltedorneBURG G., ERMert H. (2008), An Automated System for Full Angle Spatial Compounding in Ultrasound Breast Imaging, IFMBE Proc., 22, 541-545.

9. Hiller D., Ermert H. (1984), System Analysis of Ultrasound Reflection Mode Computerized Tomography, IEEE Trans. Son. Ultrason., 31, 4, 240-250.

10. Jespersen S., Wilhjelm J., Sillesen H. (1998), Multi-Angle Compound Imaging, Ultrasonic Imaging, 20, 81-102.

11. Marmarelis V.Z., Jeong J., Shin D.C., Do S. (2007), High-resolution 3-D imaging and tissue differentiation with transmission tomography in Acoustical Imaging, Springer Netherlands, 28, 195-206.

12. Nothacker M., Duda V., Hahn N., Warm M., DeGenhardt F., Madjar H., Weinbrenner S., AlBERT U. (2009), Early detection of breast cancer: benefits and risks of supplemental breast ultrasound in asymptomatic women with mammographically dense breast tissue. A systematic review, BMC Cancer, 9, 335, 1-9.

13. QuAN Y., HuAng L. (2007), Sound-speed tomography using first-arrival transmission ultrasound for a ring transducer, Medical Imaging, Proc. SPIE, 6513, 651306 .

14. Roy O., Schmidt S., Cuipin L., Allada V., West E., Kunz D., Duric N. (2013), Breast imaging using ultrasound tomography: From clinical requirements to system design, Ultrasonics Symposium (IUS), IEEE International, 1174-1177.

15. Rouyer J., Mensah S., Lasaygues P., Lefebre J.P. (2010), Ultrasound Tomography dedicated to Anatomical Breast Inspection, Proc. IEEE Ultrasonics Symposium, 2340-2343.

16. Ruiter N., ZApF M., Hopp T., DApP R., GemMEKE H. (2012), Phantom image results of an optimized full $3 D$ USCT, Medical Imaging, Proc. SPIE, 8320, 832005 .

17. Steinberg B.D. (1976), Principles of Aperture and Array System Design, John Wiley \& Sons.

18. Kino G.S. (2000), Acoustic waves, devices, imaging and analog signal processing, IEEE UFFC. 
19. Stozka R., Würfel J., Müller T., Gemmeke H. (2002), Medical Imaging by Ultrasound Computer Tomography, Medical Imaging, Proc. SPIE, 4687, 110119 .

20. Teh W., Wilson A.R.M. (1998), The role of ultrasound in breast cancer screening. A Consensus Statement by the European Group for Breast Cancer Screening, European Journal of Cancer, 34, 4, 449-450.

21. Тот T. (2011), Breast Cancer: A Lobar Disease, Springer-Verlag, London.

22. Teboul M. (1998), Imaging System for Breast Sonography, US Pat. 5, 709, 206.

23. Teboul M. (2010), Advantages of Ductal Echography (DE) over Conventional Breast Investigation in the diagnosis of breast malignancies, Medical Ultrasonography, 12, 1, 32-42.

24. WaAg R., Fedewa R. (2006), A Ring Transducer System for Medical Ultrasound Research, IEEE Trans. on UFFC, 53, 10, 1707-1718.

25. Wilhjelm J.E., Jensen M.S., Jespersen S.K., SAHL B., FAlK E. (2004), Visual and Quantitative Evaluation of Selected Image Combination Schemes in Ultrasound Spatial Compound Scanning, IEEE Trans. Med. Imag., 23, 2, 181-190.

26. Wiskin J., Borup D., Johnson S., Bergaren M., Robinson D., Smith J., Chen J., PARisky Y., KLOCK J. (2010), Inverse scattering and refraction corrected reflection for breast cancer imaging, Medical Imaging, Proc. SPIE, 7629, 7629K 1-12. 